\title{
A result of Tosio Aoki about a generalization of Hyers-Ulam stability of additive functions - a question of priority
}

\author{
LeCh MaLigranda
}

Summary. In 1940 Ulam proposed the well-known stability problem. In 1941 Hyers solved this problem for additive mappings. In 1950 Aoki proved a more general result, which was rediscovered again in 1978 by Rassias, and from then it is called by some authors the HyersUlam-Rassias (HUR) stability theorem. We show here that, in fact, this theorem merits the name Hyers-Ulam-Aoki (HUA) stability theorem.

Mathematics Subject Classification (2000). Primary 39B72, 47H15.

Keywords. Hyers-Ulam stability, Cauchy difference, inequalities, Banach spaces.

\section{On generalized Hyers-Ulam stability theorems}

Starting in November 2005 the preparation, as organizer, of the conference "International Conference Banach and Function Spaces 2006" (Sept. 14-17, 2006, Kyushu Institute of Technology, Kitakyushu, Japan) we decided that I will have 30 minutes lecture about the Japanese mathematician Tosio Aoki, who was working in the period 1946-1949 at the Meiji Technical College in Tobata which is now the Kyushu Institute of Technology, the place of the conference (cf. Maligranda [28]). Aoki is known in mathematics, mainly, for the Aoki-Rolewicz theorem (Aoki [1] proved it in 1942 and Rolewicz independently in 1957 - see [36, p. 95]) showing that a locally bounded space is $p$-convex for some $0<p \leq 1$. More precisely,

for every quasi-normed space $X=(X,\|\cdot\|)$ with the constant $C$ in the quasi-triangle inequality there is $0<p \leq 1$ and an equivalent p-norm $\|\cdot\|_{p}$ satisfying $\|x+y\|_{p}^{p} \leq\|x\|_{p}^{p}+\|y\|_{p}^{p}$ for all $x, y \in X$ such that $\|x\|_{p} \leq\|x\| \leq 2 C\|x\|_{p}$ for all $x \in X$, with $p$ satisfying $2^{1 / p-1}=C$.

As the above p-norm we can take

$$
\|x\|_{p}=\inf \left\{\left(\sum_{k=1}^{n}\left\|x_{k}\right\|^{p}\right)^{1 / p}: x=\sum_{k=1}^{n} x_{k}, n \in N\right\}
$$


(cf. Kalton-Peck-Roberts [26, p. 7] and König [27, p. 47]).

Trying to find all his published papers for a talk of 30 minutes, to be given at the beginning of the conference, it was possible to find out in the MathSciNet that Aoki published 2 mathematical papers [1], [2] and 3 papers in physics. In his second mathematical paper [2] a generalized result of Hyers [20] was proved. Here Aoki allowed growth of the form $K\left(\|x\|^{p}+\|y\|^{p}\right)$ for the norm of the Cauchy difference

$$
f(x+y)-f(x)-f(y),
$$

where $0 \leq p<1$, and still obtained the formula

$$
A(x)=\lim _{n \rightarrow \infty} \frac{f\left(2^{n} x\right)}{2^{n}}
$$

for the additive mapping approximating $f$ (this idea for the function $A$ is coming from the Hyers paper [20], where he considered the case of $p=0$ ). Let us present the Aoki result and also his proof.

Theorem 1 (Aoki Theorem, 1950). Let $X$ and $Y$ be two Banach spaces, and let $f: X \rightarrow Y$ be an "approximately additive" transformation, that is, there exists $K>0$ and $0 \leq p<1$ such that

$$
\|f(x+y)-f(x)-f(y)\| \leq K\left(\|x\|^{p}+\|y\|^{p}\right) \quad \text { for all } x, y \in X .
$$

Then there exists a unique additive function $A: X \rightarrow Y$ which is "near" $f$, i.e.,

$$
\|f(x)-A(x)\| \leq \frac{2 K}{2-2^{p}}\|x\|^{p} \quad \text { for each } \quad x \in X .
$$

Proof (Aoki [2]). By induction we can easily prove that

$$
\left\|\frac{f\left(2^{n} x\right)}{2^{n}}-f(x)\right\| \leq K \sum_{i=0}^{n-1} 2^{i(p-1)}\|x\|^{p}
$$

for any natural number $n$. The case $n=1$ follows directly from (1) with $x=y$. Assume now that (3) holds and we want to prove it for the case $n+1$. By the triangle inequality and the induction assumption (3) together with the true estimate (3) for $n=1$ we get

$$
\begin{aligned}
\left\|\frac{f\left(2^{n+1} x\right)}{2^{n+1}}-f(x)\right\| & \leq\left\|\frac{f\left(2^{n+1} x\right)}{2^{n+1}}-\frac{f(2 x)}{2}\right\|+\left\|\frac{f(2 x)}{2}-f(x)\right\| \\
& =\frac{1}{2}\left\|\frac{f\left(2^{n} 2 x\right)}{2^{n}}-f(2 x)\right\|+\left\|\frac{f(2 x)}{2}-f(x)\right\| \\
& \leq \frac{1}{2} K \sum_{i=0}^{n-1} 2^{i(p-1)}\|2 x\|^{p}+\left\|\frac{f(2 x)}{2}-f(x)\right\|
\end{aligned}
$$




$$
\begin{aligned}
& =K \sum_{i=0}^{n-1} 2^{i(p-1)} 2^{p-1}\|x\|^{p}+\left\|\frac{f(2 x)}{2}-f(x)\right\| \\
& \leq K \sum_{i=0}^{n-1} 2^{(i+1)(p-1)}\|x\|^{p}+K\|x\|^{p} \\
& =K \sum_{i=1}^{n} 2^{i(p-1)}\|x\|^{p}+K\|x\|^{p}=K \sum_{i=0}^{n} 2^{i(p-1)}\|x\|^{p},
\end{aligned}
$$

which completes the proof of (3). Since $0 \leq p<1$ it follows that

$$
\sum_{i=0}^{n-1} 2^{i(p-1)} \leq \sum_{i=0}^{\infty} 2^{i(p-1)}=\frac{1}{1-2^{p-1}}=\frac{2}{2-2^{p}}
$$

and, hence,

$$
\left\|\frac{f\left(2^{n} x\right)}{2^{n}}-f(x)\right\| \leq K_{p}\|x\|^{p}, \text { where } K_{p}=\frac{2}{2-2^{p}} K .
$$

The sequence $\left\{\frac{f\left(2^{n} x\right)}{2^{n}}\right\}$ is a Cauchy sequence in $Y$ for each $x \in X$. In fact, for $m>n>0$,

$$
\begin{aligned}
\left\|\frac{f\left(2^{m} x\right)}{2^{m}}-\frac{f\left(2^{n} x\right)}{2^{n}}\right\| & =\frac{\left\|\frac{f\left(2^{m} x\right)}{2^{m-n}}-f\left(2^{n} x\right)\right\|}{2^{n}} \\
& \leq K_{p} \frac{\left\|2^{n} x\right\|^{p}}{2^{n}}=K_{p} 2^{n(p-1)}\|x\|^{p} \rightarrow 0
\end{aligned}
$$

as $n \rightarrow \infty$. Since $Y$ is complete it follows that the sequence $\left\{\frac{f\left(2^{n} x\right)}{2^{n}}\right\}$ converges. Put

$$
A(x)=\lim _{n \rightarrow \infty} \frac{f\left(2^{n} x\right)}{2^{n}}
$$

Then

$$
\left\|f\left[2^{n}(x+y)\right]-f\left(2^{n} x\right)-f\left(2^{n} y\right)\right\| \leq K_{p}\left(\|x\|^{p}+\|y\|^{p}\right)=2^{n p} K_{p}\left(\|x\|^{p}+\|y\|^{p}\right) .
$$

Dividing both sides by $2^{n}$ and letting $n \rightarrow \infty$, we get

$$
\|A(x+y)-A(x)-A(y)\|=0 \text { or } A(x+y)=A(x)+A(y),
$$

which shows that $A$ is additive. Letting $n \rightarrow \infty$, in (3), we obtain that

$$
\|A(x)-f(x)\| \leq K_{p}\|x\|^{p}
$$

which shows that $A$ is near $f$. It remains to prove the uniqueness of $A(x)$. Let $B(x)$ be another additive transformation near $f(x)$. Then there exist $L>0$ and $0 \leq q<1$ such that

$$
\|B(x)-f(x)\| \leq L\|x\|^{q} .
$$

Hence, by (6), we have

$$
\|A(x)-B(x)\| \leq K_{p}\|x\|^{p}+L\|x\|^{q} .
$$


Therefore, in view of the additivity of $A$ and $B$,

$$
\begin{aligned}
\|A(x)-B(x)\| & =\frac{\|A(n x)-B(n x)\|}{n} \\
& \leq \frac{K_{p}\|n x\|^{p}+L\|n x\|^{q}}{n} \\
& =\frac{K_{p}\|x\|^{p}}{n^{1-p}}+\frac{L\|x\|^{q}}{n^{1-q}} \rightarrow \infty \quad \text { as } n \rightarrow \infty .
\end{aligned}
$$

We conclude that $A(x)=B(x)$.

It is a big surprise that almost nobody referred to this Aoki paper published already in 1950 but all refer to the paper by Rassias [29] from 1978. Almost thirty years after Aoki's paper, Themistocles M. Rassias [29] formulated and proved it in the same way as Aoki. He added the additional assumption about continuity of the mapping $f(t x)$ in $t$ for each fixed $x \in X$, which, as consequence, gives the linearity of $A$ not only additivity (additivity and homogeneouity of $A$ ). Thus we found it necessary to present Aoki's theorem with his proof. Only recently in the paper by Cădariu-Radu there is a stability result of Aoki-Rassias type [9] (cf. also [10], [11]), where the Aoki paper [2] is cited.

\section{Modified Hyers-Ulam stability result - a history and some results}

The stability problem appeared in 1940 when Stanisław Marcin Ulam raised the general stability problem (cf. [38], Chapter VI): "When is it true that by slightly changing the hypotheses of a theorem one can still assert that the thesis of the theorem remains true or approximately true?" or in other words: Assume that a mathematical object satisfies a certain property approximately according to some convention. Is it then possible to find an object near this object satisfying the property accurately?

In 1941 Donald H. Hyers [20] solved this problem for additive mappings with $p=0$ in Theorem 1 or in estimates (1) and (2). This result means that the additive Cauchy equation $f(x+y)=f(x)+f(y)$ is said to have "Hyers-Ulam stability on $(X, Y)$ ", where $X, Y$ are Banach spaces.

In 1945 Hyers and Ulam [22] considered the problem of stability of isometries. They asked whether there exists a constant $\kappa=\kappa(X, Y)$, depending only on $X$ and $Y$, with the property that for every surjective $\varepsilon$-isometry $T: X \rightarrow Y(T$ is $\varepsilon$ isometry if two estimates $|\|T(x)-T(y)\|-\|x-y\|| \leq \varepsilon$ for $x, y \in X$ are satisfied) there exists an isometry $U: X \rightarrow Y$ such that $\|T x-U x\| \leq \kappa \varepsilon$ for every $x \in X$. In the case where $X=Y$ and $X$ is an Euclidean or a Hilbert space, Hyers-Ulam proved that there exists an isometry $U(x)$ such that $\|T(x)-U(x)\|<10 \varepsilon$ and they used this fact to show the existence of $U(x)=\lim _{n \rightarrow \infty} T\left(2^{n} x\right) / 2^{n}$ for every $x \in X$ (see also [7], [37]). 
In 1947 Tosio Aoki [2], using the Hyers' idea, generalized his result from 1941. The Aoki paper was published in 1950 and it was received by the journal in December 29,1947 . By analogy we should say that the additive Cauchy equation has the "Hyers-Ulam-Aoki (HUA) stability on $(X, Y)$ ". This paper was overlooked and Themistocles M. Rassias [29] proved in 1978 the same result as Aoki. This result is called nowadays by some authors the "Hyers-Ulam-Rassias (HUR) stability on $(X, Y)$ ".

After discovering such unexpected observations we found it necessary to control the review articles and the books on stability results to see if the Aoki paper is cited somewhere. It is again a big surprise that the "paper was NOT cited", and the conclusions are the following:

(i) The reviewer for the Mathematical Rewievs of the Aoki paper [2] was David Gordon Bourgin [see MR0040580 (12,717a)] and he pointed out that Hyers' method effects the proof of Aoki and that he himself has shown the right sides of (1) and (2) cannot be replaced by the condition that the limsup, as $\|x\|$ and $\|y\| \rightarrow \infty$, is 0 when $p=1$ (to be precise the left side divided by the right side in (1) and (2)). His example was published in [6, p. 224].

Moreover, Bourgin in his paper [6] published in 1951 didn't refer to Aoki [2] and also not in his later papers. Let us note that Bourgin, in an other paper [4], sharpened a result of Hyers [19] by showing that a locally bounded space is given by a quasi-norm. Lately Aoki, in paper [1], proved that these two properties are equivalent to the existence of an $p$-homogeneous norm on the space.

(ii) The reviewer for Zentralblatt (Zbl 0040.35501) was Alfredo Pereira Gomes from Portugal, who described there Aoki's theorem (Theorem 1) as a generalization of the Hyers result but he was not publishing anything else neither in the stability theory nor in the theory of functional equations.

(iii) In 1978 the above mentioned paper of Rassias [29] appeared. He proved the theorem, which is almost exactly the same as Aoki's result, adding only the continuity and having in conclusion the linearity of $A$.

Remark 1. The arguments above show that the generalized Hyers-Ulam stability result proved by Rassias in 1978 was proved already in 1950 by Aoki.

(iv) In 1990 Rassias ([30], p. 309) noticed that the proof of Theorem 1 also works for $p<0$ and asked about the case $p \geq 1$. In 1991, Gajda [16] extended Theorem 1 to the case $p>1$. His idea to prove the theorem was to replace $n$ by $-n$ in the formula (5), that is, the additive mapping $A$ is given by the formula $A(x)=\lim _{n \rightarrow \infty} 2^{n} f\left(\frac{x}{2^{n}}\right)$ and which as a consequence give in (2) the constant $\frac{2 K}{2^{p}-2}$. He also gave a counterexample showing that the stability theorem is false for $p=1$, for any choice of $K>0$. His function was the following: $f: R \rightarrow R$ given by the formula $f(x)=\sum_{n=0}^{\infty} \frac{\varphi\left(2^{n} x\right)}{2^{n}}$, where

$$
\varphi(x)=\frac{-K}{6} \chi_{(-\infty, 1]}(x)+\frac{K x}{6} \chi_{(-1,1)}(x)+\frac{K}{6} \chi_{(1, \infty)}(x)
$$


(cf. also [31], pp. 32-33). In 1992 Rassias and Šemrl [33] showed that the function $f: \mathbb{R} \rightarrow \mathbb{R}$ given by

$$
f(x)=x \log _{2}|x-1| \chi_{(-\infty, 0)}(x)+x \log _{2}(x+1) \chi_{[0, \infty)}(x)
$$

satisfies (1) with $p=1$ and $|f(x)-c x| /|x| \rightarrow \infty$ as $x \rightarrow \infty$, for any real number $c$. In 2001 Găvruța [18] proved that the function $f(x)=x \ln |x|$, if $x \neq 0$ and $f(0)=0$ satisfies (1) with $K=p=1$ but

$$
\sup _{x \neq 0} \frac{|f(x)-A(x)|}{|x|} \geq \sup _{n \in N} \frac{|n \ln n-A(n)|}{n}=\sup _{n \in N}|\ln n-A(1)|=\infty
$$

for any additive function $A: R \rightarrow R$. Moreover, Brzdȩk [8] observed that for $p>0, p \neq 1$ the constant $2 /\left|2^{p}-2\right|$ in estimate (2) is sharp. All the above results we can also find in the Rassias paper [31] and in the book by Jung [24, Section 1.3].

Remark 2. The Hyers-Ulam-Aoki stability result holds for any $p \in \mathbb{R} \backslash\{1\}$.

(v) The sequence $\left\{\frac{f\left(2^{n} x\right)}{2^{n}}\right\}$ is generally called Hyers sequence, because Hyers started for the first time the direct method, where the mapping $A$ is explicitly constructed by the formula (5).

(vi) Bourgin [6, p. 224] replaced the constant $\varepsilon$ in the control relation (1) in Hyers' theorem [20] (that is the case $p=0$ and $K=\varepsilon / 2$ in Aoki's Theorem 1) by a function $\psi(x, y)$, a positive monotone nondecreasing symmetric function of $\|x\|$ and $\|y\|$ such that, for every $x \in X, \mu(x):=\sum_{k=1}^{\infty} 2^{-k} \psi\left(2^{k}\|x\|, 2^{k}\|x\|\right)$ is convergent, and still obtained a stability result with the estimate $\|f(x)-A(x)\|$ $\leq \mu(x)$ for all $x \in X$. On the other hand, a general stability result for a large class of functional equations of the form $f(G(x, y))=H(f(x), f(y))$, with suitable conditions for $G$ and $H$ has been proved by Forti in [14]. Later on Găvrută [17] also gave a stability result of Bourgin type by considering as control mapping $\varphi: G \times G \rightarrow[0, \infty)$, which satisfies $\sum_{k=0}^{\infty} 2^{(k+1)} \varphi\left(2^{k} x, 2^{k} y\right)<$ $\infty$ for all $x, y \in G$ and $G$ is an Abelian group.

For related results see Rassias-Šemrl [34] and Borelli-Forti [3]. Let us mention that there are also generalized Hyers-Ulam stability results on restricted domains (cf. [21], [31, pp. 52-56] and Jung [24, Th. 1.11]).

(vii) The following survey papers, containing historical informations and a lot of references, were published: in 1995 by Gian Luigi Forti [15], in 1997 by SoonMo Jung [23], in 2000 by Themistocles M. Rassias [31], [32] and in 2003 by Soon-Mo Jung [25], but there is nothing there about the Aoki result from 1950.

(viii) There are monographs on the stability theory of the Hyers-Ulam-Rassias type with historical backgrounds and important results: Hyers-Isac-Rassias [21, Chapter 2] published in 1998, Jung [24, Section 1.3] published in 2001, Czerwik [12, Chapter 13] published in 2002 but also here there is nothing written about the Aoki result. 
The book [13] is even dedicated to the Twenty Fifth anniversary of the publication of Rassias [29], which had some influence in the development of the stability of functional equations.

We finish our discussion with the following problem:

Problem. Why Aoki's paper published in 1950 was completely forgotten or overlooked and all mathematicians refer only to the Rassias paper from 1978?

Acknowledgements. I would like to thank first referee for the references [9], [10], [11], [14] and xerox-copy of the paper [9] (I didn't see papers [10] and [11]). I thank the second referee for information about papers [14], [3] and both referees for the corrections of remark (vi). I would like to thank also the Editors for the suggestion to change my original title "Hyers-Ulam-Aoki stability theorem rather than Hyers-Ulam-Rassias stability theorem - a question of priority" to the one which is now.

\section{References}

[1] T. Aoki, Locally bounded linear topological spaces, Proc. Imp. Acad. Tokyo 18 (1942), $588-594$.

[2] T. Aoki, On the stability of the linear transformation in Banach spaces, J. Math. Soc. Japan 2 (1950), 64-66.

[3] C. Borelli and G. L. Forti, On a general Hyers-Ulam stability result, Internat. J. Math. Math. Sci. 18 (1995), no. 2, 229-236.

[4] D. G. Bourgin, Linear topological spaces, Amer. J. Math. 65 (1943), 637-659.

[5] D. G. Bourgin, Approximate isometries, Bull. Amer. Math. Soc. 52 (1946), 704-714.

[6] D. G. Bourgin, Classes of transformations and bordering transformations, Bull. Amer. Math. Soc. 57 (1951), 223-237.

[7] R. G. Bourgin, Approximate isometries on finite dimensional Banach spaces, Trans. Amer. Math. Soc. 207 (1975), 309-328.

[8] J. BRZDȨK, A note on stability of additive mappings, in: Stability of mappings of HyersUlam type, 19-22, Hadronic Press, Palm Harbor, 1994.

[9] L. CĂDARIU and V. RADU, Stability properties of monomial functional equations, An. Univ. Timişoara Ser. Mat.-Inform. 43 (2005), no. 1, 1-19.

[10] L. CĂDARIU and V. RADU, Stability results for a functional equation of quartic type, Automat. Comput. Appl. Math. 15 (2006), no. 1, 7-21.

[11] L. CÃDARIU and V. RADU, The alternative of fixed point and stability results for functional equations, Int. J. Appl. Math. Stat. (2007), to appear in a volume dedicated to Euler.

[12] S. Czerwik, Functional Equations and Inequalities in Several Variables, World Scientific, New Jersey, 2002.

[13] S. Czerwik (ed.), Stability of Functional Equations of Ulam-Hyers-Rassias Type, Hadronic Press, Palm Harbor, Florida, 2003.

[14] G. L. ForTi, An existence and stability theorem for a class of functional equations, Stochastica 4 (1980), no. 1, 23-30.

[15] G. L. ForTI, Hyers-Ulam stability of functional equations in several variables, Aequationes Math. 50 (1995), No. 1-2, 143-190.

[16] Z. GajdA, On stability of additive mappings, Internat. J. Math. Sci. 14 (1991), No. 3, 431-434. 
[17] P. Găvrută, A generalization of the Hyers-Ulam-Rassias stability of approximately additive mappings, J. Math. Anal. Appl. 184 (1994), No. 3, 431-436.

[18] P. GĂVRUTĂ, On a problem of G. Isac and Th. M. Rassias concerning the stability of mappings, J. Math. Anal. Appl. 261 (2001), 543-553.

[19] D. H. Hyers, Locally bounded linear topological spaces, Revista Ci., Lima 41 (1939), 555574 .

[20] D. H. Hyers, On the stability of the linear functional equation, Proc. Nat. Acad. Sci. USA 27 (1941), 222-224; reprinted in [35], 165-168.

[21] D. H. Hyers, G. Isac and T. M. Rassias, Stability of Functional Equations in Several Variables, Birkhäuser, Boston, 1998.

[22] D. H. Hyers and S. M. Ulam, On approximate isometries, Bull. Amer. Math. Soc. 51 (1945), 288-292.

[23] S.-M. Jung, Hyers-Ulam-Rassias stability of functional equations, Dynam. Systems Appl. 6 (1997), No. 4, 541-565.

[24] S.-M. JUng, Hyers-Ulam-Rassias Stability of Functional Equations in Mathematical Analysis, Hadronic Press, Inc., Palm Harbor, 2001.

[25] S.-M. Jung, Survey on the Hyers-Ulam-Rassias stability of functional equations, in: S. Czerwik (ed.), Stability of Functional Equations of Ulam-Hyers-Rassias Type, 91-117, Hadronic Press, Inc., Palm Harbor, 2001.

[26] N. J. Kalton, N. T. Peck and J. W. Roberts, An F-space Sampler, Cambridge Univ. Press, Cambridge, 1984.

[27] H. KÖNIG, Eigenvalue Distribution of Compact Operators, Birkhäuser Verlag, Basel, 1986

[28] L. Maligranda, Tosio Aoki (1910-1989), Lecture which was presented on the 14th September 2006 at the conference "Banach and Function Spaces 2006", Sept. 14-17, 2006, Kyushu Institute of Technology, Kitakyushu, Japan (it will be published in the conference proceedings).

[29] Тн. M. Rassias, On the stability of the linear mapping in Banach spaces, Proc. Amer. Math. Soc. 72 (1978), No. 2, 297-300.

[30] TH. M. Rassias, The stability of mappings and related topics, in: Report on the 27th Internat. Symp. on Functional Equations, Aequationes Math. 39 (1990), 292-293, 309.

[31] Тн. M. Rassias, On the stability of functional equations and a problem of Ulam, Acta Appl. Math. 62 (2000), No. 1, 23-130.

[32] TH. M. Rassias, On the stability of functional equations in Banach spaces, J. Math. Anal. Appl. 251 (2000), No. 1, 264-284.

[33] Th. M. Rassias and P. ŠEmRL, On the behavior of mappings which do not satisfy HyersUlam stability, Proc. Amer. Math. Soc. 114 (1992), No. 4, 989-993.

[34] Th. M. Rassias and P. SEmRl, On the Hyers-Ulam stability of linear mappings, J. Math. Anal. Appl. 173 (1993), 325-338.

[35] Th. M. Rassias and J. TABOR (eds.), Stability of Mappings of Hyers-Ulam Type, Hadronic Press Collection of Original Articles, Hadronic Press, Palm Harbor, 1994.

[36] S. Rolewicz, Metric Linear Spaces, PWN, Warsaw, 1972.

[37] P. Šemrl, Hyers-Ulam stability of isometries on Banach spaces, Aequationes Math. 58 (1999), No. 1-2, 157-162.

[38] S. M. Ulam, A Collection of Mathematical Problems, Interscience Tracts in Pure and Applied Mathematics, No. 8, Interscience Publishers, New York-London, 1960.

L. Maligranda

Department of Mathematics

Luleå University of Technology

SE-971 87 Luleå

Sweden

e-mail: lech@sm.luth.se

Manuscript received: August 29, 2006 and, in final form, January 26, 2007. 